\title{
Multiple positive solutions of semilinear elliptic equations involving concave and convex nonlinearities in $\mathbb{R}^{N}$
}

Huei-li Lin

Correspondence: hlin@mail.cgu. edu.tw

Department of Natural Sciences in the Center for General Education, Chang Gung University, Tao-Yuan 333, Taiwan

\section{Abstract}

In this article, we investigate the effect of the coefficient $f(z)$ of the sub-critical nonlinearity. For sufficiently large $\lambda>0$, there are at least $k+1$ positive solutions of the semilinear elliptic equations

$$
\left\{\begin{array}{l}
-\Delta v+\lambda v=f(z) v^{p-1}+h(z) v^{q-1} \text { in } \mathbb{R}^{N} \\
v \in H^{1}\left(\mathbb{R}^{N}\right)
\end{array}\right.
$$

where $1 \leq q<2<p<2^{*}=2 N /(N-2)$ for $N \geq 3$.

AMS (MOS) subject classification: 35J20; 35J25; 35J65.

Keywords: semilinear elliptic equations, concave and convex, positive solutions

\section{Introduction}

For $N \geq 3,1 \leq q<2<p<2^{*}=2 N /(N-2)$, we consider the semilinear elliptic equations

$$
\left\{\begin{array}{l}
-\Delta v+\lambda v=f(z) v^{p-1}+h(z) v^{q-1} \text { in } \mathbb{R}^{N} \\
v \in H^{1}\left(\mathbb{R}^{N}\right)
\end{array}\right.
$$

where $\lambda>0$.

Let $f$ and $h$ satisfy the following conditions:

( $f$ 1) $f$ is a positive continuous function in $\mathbb{R}^{N}$ and $\lim _{|z|} \rightarrow \infty f(z)=f_{\infty}>0$.

(f2) there exist $k$ points $a^{1}, a^{2}, \ldots, a^{k}$ in $\mathbb{R}^{N}$ such that

$$
f\left(a^{i}\right)=f_{\max }=\max _{z \in \mathbb{R}^{N}} f(z) \text { for } 1 \leq \mathrm{i} \leq k,
$$

and $f_{\infty}<f_{\max }$.

(h 1) $h \in L^{\frac{p}{p-q}}\left(\mathbb{R}^{N}\right) \cap L^{\infty}\left(\mathbb{R}^{N}\right)$ and $h \supsetneqq 0$.

Semilinear elliptic problems involving concave-convex nonlinearities in a bounded domain

$$
\left\{\begin{array}{l}
-\Delta u=\operatorname{ch}(z)|u|^{q-2} u+|u|^{p-2} u \text { in } \Omega \\
u \in H_{0}^{1}(\Omega)
\end{array}\right.
$$


have been studied by Ambrosetti et al. [1] $\left(h \equiv 1\right.$, and $\left.1<q<2<p \leq 2^{*}=2 N /(N-2)\right)$ and Wu $[2] h \in C(\bar{\Omega})$ and changes sign, $\left.1<q<2<p<2^{*}\right)$. They proved that this equation has at least two positive solutions for sufficiently small $c>0$. More general results of Equation $\left(E_{c}\right)$ were done by Ambrosetti et al. [3], Brown and Zhang [4], and de Figueiredo et al. [5].

In this article, we consider the existence and multiplicity of positive solutions of Equation $\left(E_{\lambda}\right)$ in $\mathbb{R}^{N}$. For the case $q=\lambda=1$ and $f(z) \equiv 1$ for all $z \in \mathbb{R}^{N}$, suppose that $h$ is nonnegative, small, and exponential decay, Zhu [6] showed that Equation $\left(E_{\lambda}\right)$ admits at least two positive solutions in $\mathbb{R}^{N}$. Without the condition of exponential decay, Cao and Zhou [7] and Hirano [8] proved that Equation $\left(E_{\lambda}\right)$ admits at least two positive solutions in $\mathbb{R}^{N}$. For the case $q=\lambda=1$, by using the idea of category and Bahri-Li's minimax argument, Adachi and Tanaka [9] asserted that Equation $\left(E_{\lambda}\right)$ admits at least four positive solutions in $\mathbb{R}^{N}$, where $f(z) \otimes 1, f(z) \geq 1-C \exp ((-(2+\delta)|z|)$ for some $C$, $\delta>0$, and sufficiently small $\|h\|_{H^{-1}}>0$. Similarly, in Hsu and Lin [10], they have studied that there are at least four positive solutions of the general case $-\Delta u+u=f(z) v^{p-1}$ $+\lambda h(z) v^{q-1}$ in $\mathbb{R}^{N}$ for sufficiently small $\lambda>0$.

By the change of variables

$$
\varepsilon=\lambda^{-\frac{1}{2}} \text { and } u(z)=\varepsilon^{\frac{2}{p-2}} v(\varepsilon z),
$$

Equation $\left(E_{\lambda}\right)$ is transformed to

$$
\left\{\begin{array}{l}
-\Delta u+u=f(\varepsilon z) u^{p-1}+\varepsilon^{\frac{2(p-q)}{p-2}} h(\varepsilon z) u^{q-1} \text { in } \mathbb{R}^{N} \\
u \in H^{1}\left(\mathbb{R}^{N}\right)
\end{array}\right.
$$

Associated with Equation $\left(E_{\varepsilon}\right)$, we consider the $C^{1}$-functional $J_{\varepsilon}$, for $u \in H^{1}\left(\mathbb{R}^{N}\right)$,

$$
J_{\varepsilon}(u)=\frac{1}{2}\|u\|_{H}^{2}-\frac{1}{p} \int_{\mathbb{R}^{N}} f(\varepsilon z) u_{+}^{p} d z-\frac{1}{q} \int_{\mathbb{R}^{N}} \varepsilon^{\frac{2(p-q)}{p-2}} h(\varepsilon z) u_{+}^{q} d z,
$$

where $\|u\|_{H}^{2}=\int_{\mathbb{R}^{N}}\left(|\Delta u|^{2}+|u|^{2}\right) d z$ is the norm in $H^{1}\left(\mathbb{R}^{N}\right)$ and $u_{+}=\max \{u, 0\} \geq 0$. We know that the nonnegative weak solutions of Equation $\left(E_{\varepsilon}\right)$ are equivalent to the critical points of $J_{\varepsilon}$. This article is organized as follows. First of all, we use the argument of Tarantello [11] to divide the Nehari manifold $\mathbf{M}_{\varepsilon}$ into the two parts $\mathbf{M}_{\varepsilon}^{+}$and $\mathbf{M}_{\varepsilon}^{-}$. Next, we prove that the existence of a positive ground state solution $u_{0} \in \mathbf{M}_{\varepsilon}^{+}$of Equation $\left(E_{\varepsilon}\right)$. Finally, in Section 4, we show that the condition $(f 2)$ affects the number of positive solutions of Equation $\left(E_{\varepsilon}\right)$, that is, there are at least $k$ critical points $u_{1}, \ldots, u_{k} \in \mathbf{M}_{\varepsilon}^{-}$ of $J_{\varepsilon}$ such that $J_{\varepsilon}\left(u_{i}\right)=\beta_{\varepsilon}^{i}((\mathrm{PS})-$ value $)$ for $1 \leq i \leq k$.

Let

$$
S=\sup _{\substack{u \in H^{1}\left(\mathbb{R}^{N}\right) \\\|u\|_{H^{\prime}=1}}}\|u\|_{L^{p}}
$$

then

$$
\|u\|_{L^{p}} \leq S\|u\|_{H} \quad \text { for any } u \in H^{1}\left(\mathbb{R}^{N}\right) \backslash\{0\} .
$$


For the semilinear elliptic equations

$$
\left\{\begin{array}{l}
-\Delta u+u=f(\varepsilon z) u^{p-1} \text { in } \mathbb{R}^{N} \\
u \in H^{1}\left(\mathbb{R}^{N}\right)
\end{array}\right.
$$

we define the energy functional $I_{\varepsilon}(u)=\frac{1}{2}\|u\|_{H}^{2}-\frac{1}{p} \int_{\mathbb{R}^{N}} f(\varepsilon z) u_{+}^{p} d z$, and

$$
\gamma_{\varepsilon}=\inf _{u \in \mathbf{N}_{\varepsilon}} I_{\varepsilon}(u),
$$

where $\mathbf{N}_{\varepsilon}=\left\{u \in H^{1}\left(\mathbb{R}^{N}\right) \backslash\{0\} \mid u_{+} \otimes 0\right.$ and $\left.\left\langle I_{\varepsilon}^{\prime}(u), u\right\rangle=0\right\}$. Note that

(i) if $f \equiv f_{\infty}$, we define $I_{\infty}(u)=\frac{1}{2}\|u\|_{H}^{2}-\frac{1}{p} \int_{\mathbb{R}^{N}} f_{\infty} u_{+}^{p} d_{z}$ and

$$
\gamma_{\infty}=\inf _{u \in \mathbf{N}_{\infty}} I_{\infty}(u)
$$

where $\mathbf{N}_{\infty}=\left\{u \in H^{1}\left(\mathbb{R}^{N}\right) \backslash\{0\} \mid u_{+} \otimes 0\right.$ and $\left.\left\langle I_{\infty}^{\prime}(u), u\right\rangle=0\right\}$;

(ii) if $f \equiv f_{\max }$, we define $I_{\max }(u)=\frac{1}{2}\|u\|_{H}^{2}-\frac{1}{p} \int_{\mathbb{R}^{N}} f_{\max } u_{+}^{p} d z$ and

$$
\gamma_{\max }=\inf _{u \in \mathbf{N}_{\max }} I_{\max }(u),
$$

where $\mathbf{N}_{\max }=\left\{u \in H^{1}\left(\mathbb{R}^{N}\right) \backslash\{0\} \mid u_{+} \otimes 0\right.$ and $\left.\left\langle I_{\max }^{\prime}(u), u\right\rangle=0\right\}$.

Lemma 1.1

$$
\gamma_{\max }=\frac{p-2}{2 p}\left(f_{\max } S^{p}\right)^{-2 /(p-2)}>0 .
$$

Proof. It is similar to Theorems 4.12 and 4.13 in Wang [[12], p. 31].

Our main results are as follows.

(I) Let $\Lambda=\varepsilon^{2(p-q) /(p-2)}$. Under assumptions $(f 1)$ and $(h 1)$, if

$$
0<\Lambda<\Lambda_{0}=(p-2)\left(\frac{2-q}{f_{\max }}\right)^{\frac{2-q}{p-2}}\left[(p-q) S^{2}\right]^{\frac{q-p}{p-2}}\|h\|_{\#}^{-1},
$$

where $\|h\|_{\# \text { is the norm in } \frac{p}{p-q}\left(\mathbb{R}^{N}\right)}$, then Equation $\left(E_{\varepsilon}\right)$ admits at least a positive ground state solution. (See Theorem 3.4)

(II) Under assumptions (f1) - (f2) and ( $h 1)$, if $\lambda$ is sufficiently large, then Equation $\left(E_{\lambda}\right)$ admits at least $k+1$ positive solutions. (See Theorem 4.8)

\section{The Nehari manifold}

First of all, we define the Palais-Smale (denoted by (PS)) sequences and (PS)-conditions in $H^{1}\left(\mathbb{R}^{N}\right)$ for some functional $J$.

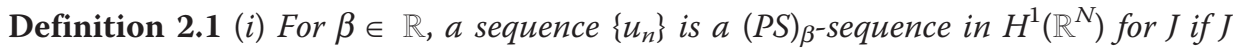
$\left(u_{n}\right)=\beta+o_{n}(1)$ and $J\left(u_{n}\right)=o_{n}(1)$ strongly in $H^{-1}\left(\mathbb{R}^{N}\right)$ as $n \rightarrow \infty$, where $H^{-1}\left(\mathbb{R}^{N}\right)$ is the dual space of $H^{1}\left(\mathbb{R}^{N}\right)$; 


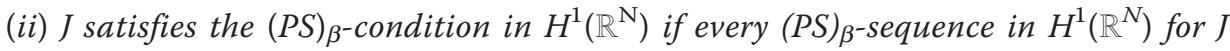
contains a convergent subsequence.

Next, since $J_{\varepsilon}$ is not bounded from below in $H^{1}\left(\mathbb{R}^{N}\right)$, we consider the Nehari manifold

$$
\mathbf{M}_{\varepsilon}=\left\{u \in H^{1}\left(\mathbb{R}^{N}\right) \backslash\{0\} \mid u_{+} \not \equiv 0 \text { and }\left\langle J_{\varepsilon}^{\prime}(u), u\right\rangle=0\right\},
$$

where

$$
\left\langle J_{\varepsilon}^{\prime}(u), u\right\rangle=\|u\|_{H}^{2}-\int_{\mathbb{R}^{N}} f(\varepsilon z) u_{+}^{p} d z-\int_{\mathbb{R}^{N}} \varepsilon^{\frac{2(p-q)}{p-2}} h(\varepsilon z) u_{+}^{q} d z .
$$

Note that $\mathbf{M}_{\varepsilon}$ contains all nonnegative solutions of Equation $\left(E_{\varepsilon}\right)$. From the lemma below, we have that $J_{\varepsilon}$ is bounded from below on $\mathbf{M}_{\varepsilon}$.

Lemma 2.2 The energy functional $J_{\varepsilon}$ is coercive and bounded from below on $\mathbf{M}_{\varepsilon}$.

Proof. For $u \in \mathbf{M}_{\varepsilon}$, by (2.1), the Hölder inequality $\left(p_{1}=\frac{p}{p-q}, p_{2}=\frac{p}{q}\right)$ and the Sobolev embedding theorem (1.1), we get

$$
\begin{aligned}
J_{\varepsilon}(u) & =\left(\frac{1}{2}-\frac{1}{p}\right)\|u\|_{H}^{2}-\left(\frac{1}{q}-\frac{1}{p}\right) \int_{\mathbb{R}^{N}} \frac{2(p-q)}{p-2} h(\varepsilon z) u_{+}^{q} d z \\
& \geq \frac{\|u\|_{H}^{q}}{p}\left[\frac{p-2}{2}\|u\|_{H}^{2-q}-\frac{p-q}{q} \varepsilon \frac{2(p-q)}{p-2}\|h\|_{\#} S^{q}\right] .
\end{aligned}
$$

Hence, we have that $J_{\varepsilon}$ is coercive and bounded from below on $\mathbf{M}_{\varepsilon}$.

Define

$$
\psi_{\varepsilon}(u)=\left\langle J_{\varepsilon}^{\prime}(u), u\right\rangle .
$$

Then for $u \in \mathbf{M}_{\varepsilon}$, we get

$$
\begin{aligned}
& \left\langle\psi_{\varepsilon}^{\prime}{ }_{\varepsilon}(u), u\right\rangle=2\|u\|_{H}^{2}-p \int_{\mathbb{R}^{N}} f(\varepsilon z) u_{+}^{p} d z-q \int_{\mathbb{R}^{N}} \varepsilon^{\frac{2(p-q)}{p-2}} h(\varepsilon z) u_{+}^{q} d z \\
& =(p-q) \int_{\mathbb{R}^{N}} \varepsilon^{\frac{2(p-q)}{p-2}} h(\varepsilon z) u_{+}^{q} d z-(p-2)\|u\|_{H}^{2} \\
& =(2-q)\|u\|_{H}^{2}-(p-q) \int_{\mathbb{R}^{N}} f(\varepsilon z) u_{+}^{p} d z .
\end{aligned}
$$

We apply the method in Tarantello [11], let

$$
\begin{aligned}
\mathbf{M}_{\varepsilon}^{+} & =\left\{u \in \mathbf{M}_{\varepsilon} \mid\left\langle\psi_{\varepsilon}^{\prime}(u), u\right\rangle>0\right\} ; \\
\mathbf{M}_{\varepsilon}^{0} & =\left\{u \in \mathbf{M}_{\varepsilon} \mid\left\langle\psi_{\varepsilon}^{\prime}(u), u\right\rangle=0\right\} ; \\
\mathbf{M}_{\varepsilon}^{-} & =\left\{u \in \mathbf{M}_{\varepsilon} \mid\left\langle\psi_{\varepsilon}^{\prime}(u), u\right\rangle<0\right\} .
\end{aligned}
$$


Lemma 2.3 Under assumptions $(f 1)$ and $(h 1)$, if $0<\Lambda\left(=\varepsilon^{2(p-q) /(p-2)}\right)<\Lambda_{0}$, then $\mathbf{M}_{\varepsilon}^{0}=\emptyset$.

Proof. See Hsu and Lin [[10], Lemma 5].

Lemma 2.4 Suppose that $u$ is a local minimizer for $J_{\varepsilon}$ on $\mathbf{M}_{\varepsilon}$ and $u \notin \mathbf{M}_{\varepsilon}^{0}$. Then $J_{\varepsilon}^{\prime}(u)=\operatorname{Oin} H^{-1}\left(\mathbb{R}^{N}\right)$.

Proof. See Brown and Zhang [[4], Theorem 2.3].

Lemma 2.5 We have the following inequalities.

(i) $\int_{\mathbb{R}^{N}} h(\varepsilon z) u_{+}^{q} d z>0_{\text {for each }} u \in \mathbf{M}_{\varepsilon}^{+}$;

(ii) $\|u\|_{H}<\left(\frac{p-q}{p-2} \Lambda\|h\|_{\#} S^{q}\right)^{1 /(2-q)}$ for each $u \in \mathbf{M}_{\varepsilon}^{+}$;

(iii) $\|u\|_{H}>\left[\frac{2-q}{(p-q) f_{\max } S^{p}}\right]^{1 /(p-2)}$ for each $u \in \mathbf{M}_{\varepsilon}^{-}$;

(iv) If $0<\Lambda\left(=\varepsilon^{2(p-q) /(p-2)}\right)<\frac{q \Lambda_{0}}{2}$, then $J_{\varepsilon}(u)>0$ for each $u \in \mathbf{M}_{\varepsilon}^{-}$.

Proof. (i) It can be proved by using (2.2).

(ii) For any $u \in \mathbf{M}_{\varepsilon}^{+} \subset \mathbf{M}_{\varepsilon}$, by (2.2), we apply the Hölder inequality $\left(p_{1}=\frac{p}{p-q}, p_{2}=\frac{p}{q}\right)$ to obtain that

$$
\begin{aligned}
0 & <(p-q) \int_{\mathbb{R}^{N}} \Lambda h(\varepsilon z) u_{+}^{q} d z-(p-2)\|u\|_{H}^{2} \\
& \leq(p-q) \Lambda\|h\|_{\#} S^{q}\|u\|_{H}^{q}-(p-2)\|u\|_{H}^{2} .
\end{aligned}
$$

(iii) For any $u \in \mathbf{M}_{\varepsilon}^{-}$, by (2.3), we have that

$$
\|u\|_{H}^{2}<\frac{p-q}{2-q} \int_{\mathbb{R}^{N}} f(\varepsilon z) u_{+}^{p} d z \leq \frac{p-q}{2-q} S^{p}\|u\|_{H}^{p} f_{\max } .
$$

(iv) For any $u \in \mathbf{M}_{\varepsilon}^{-} \subset \mathbf{M}_{\varepsilon}$, by (iii), we get that

$$
\begin{aligned}
J_{\varepsilon}(u) & =\left(\frac{1}{2}-\frac{1}{p}\right)\|u\|_{H}^{2}-\left(\frac{1}{q}-\frac{1}{p}\right) \int_{\mathbb{R}^{N}} \Lambda h(\varepsilon z) u_{+}^{q} d z \\
& \geq \frac{\|u\|_{H}^{q}}{p}\left[\frac{p-2}{2}\|u\|_{H}^{2-q}-\frac{p-q}{q} \Lambda\|h\|_{\#} S^{q}\right] \\
& >\frac{1}{p}\left[\frac{2-q}{(p-q) f_{\max } S^{p}}\right]^{\frac{q}{p-2}}\left[\frac{p-2}{2}\left[\frac{2-q}{(p-q) f_{\max } S^{p}}\right]^{\frac{2-q}{p-2}}-\frac{p-q}{q} \Lambda\|h\|_{\#} S^{q}\right] .
\end{aligned}
$$

Thus, if $0<\Lambda<\frac{q}{2}(p-2)\left(\frac{2-q}{f_{\max }}\right)^{\frac{2-q}{p-2}}\left[(p-q) S^{2}\right]^{\frac{q-p}{p-2}}\|h\|_{\#}^{-1}$, we get that $J_{\varepsilon}(u) \geq$ $d_{0}>0$ for some constant $d_{0}=d_{0}\left(\varepsilon, p, q, S,\|h\|_{\#}, f_{\max }\right)$.

For $u \in H^{1}\left(\mathbb{R}^{N}\right) \backslash\{0\}$ and $u_{+} \otimes 0$, let

$$
\bar{t}=\bar{t}(u)=\left[\frac{(2-q)\|u\|_{H}^{2}}{(p-q) \int_{\mathbb{R}^{N}} f(\varepsilon z) u_{+}^{p} d z}\right]^{1 /(p-2)}>0 .
$$


Lemma 2.6 For each $u \in H^{1}\left(\mathbb{R}^{N}\right) \backslash\{0\}$ and $u_{+} \otimes 0$, we have that

(i) if $\int_{\mathbb{R}^{N}} h(\varepsilon z) u_{+}^{q} d z=0$, then there exists a unique positive number $t^{-}=t^{-}(u)>\bar{t}$ such that $t^{-} u \in \mathbf{M}_{\varepsilon}^{-}$and $J_{\varepsilon}\left(t^{-} u\right)=\sup _{t \geq 0} J_{\varepsilon}(t u)$;

(ii) if $0<\Lambda\left(=\varepsilon^{2(p-q) /(p-2)}\right)<\Lambda_{0}$ and $\int_{\mathbb{R}^{N}} h(\varepsilon z) u_{+}^{q} d z>0$, then there exist unique positive numbers $t^{+}=t^{+}(u)<\bar{t}<t^{-}=t^{-}(u)$ such that $t^{+} u \in \mathbf{M}_{\varepsilon}^{+}, t^{-} u \in \mathbf{M}_{\varepsilon}^{-}$and

$$
J_{\varepsilon}\left(t^{+} u\right)=\inf _{0 \leq t \leq \bar{t}} J_{\varepsilon}(t u), \quad J_{\varepsilon}\left(t^{-} u\right)=\sup _{t \geq \bar{t}} J_{\varepsilon}(t u) .
$$

Proof. See Hsu and Lin [[10], Lemma 7].

Applying Lemma $2.3\left(\mathbf{M}_{\varepsilon}^{0}=\emptyset\right.$ for $\left.0<\Lambda<\Lambda_{0}\right)$, we write $\mathbf{M}_{\varepsilon}=\mathbf{M}_{\varepsilon}^{+} \cup \mathbf{M}_{\varepsilon}^{-}$, where

$$
\begin{aligned}
& \mathbf{M}_{\varepsilon}^{+}=\left\{u \in \mathbf{M}_{\varepsilon} \mid(2-q)\|u\|_{H}^{2}-(p-q) \int_{\mathbb{R}^{N}} f(\varepsilon z) u_{+}^{p} d z>0\right\}, \\
& \mathbf{M}_{\varepsilon}^{-}=\left\{u \in \mathbf{M}_{\varepsilon} \mid(2-q)\|u\|_{H}^{2}-(p-q) \int_{\mathbb{R}^{N}} f(\varepsilon z) u_{+}^{p} d z<0\right\} .
\end{aligned}
$$

Define

$$
\alpha_{\varepsilon}=\inf _{u \in \mathbf{M}_{\varepsilon}} J_{\varepsilon}(u) ; \quad \alpha_{\varepsilon}^{+}=\inf _{u \in \mathbf{M}_{\varepsilon}^{+}} J_{\varepsilon}(u) ; \quad \alpha_{\varepsilon}^{-}=\inf _{u \in \mathbf{M}_{\varepsilon}^{-}} J_{\varepsilon}(u) .
$$

Lemma 2.7 (i) If $0<\Lambda\left(=\varepsilon^{2(p-q) /(p-2)}\right)<\Lambda_{0}$, then $\alpha_{\varepsilon} \leq \alpha_{\varepsilon}^{+}<0$;

(ii) If $0<\Lambda<q \Lambda_{0} / 2$, then $\alpha_{\varepsilon}^{-} \geq d_{0}>$ Ofor some constant $d_{0}=d_{0}\left(\varepsilon, p, q, S,\|h\|_{\#,} f_{\max }\right)$.

Proof. (i) Let $u \in \mathbf{M}_{\varepsilon}^{+}$, by (2.2), we get

$$
(p-2)\|u\|_{H}^{2}<(p-q) \int_{\mathbb{R}^{N}} \Lambda h(\varepsilon z) u_{+}^{q} d z .
$$

Then

$$
\begin{aligned}
J_{\varepsilon}(u) & =\left(\frac{1}{2}-\frac{1}{p}\right)\|u\|_{H}^{2}-\left(\frac{1}{q}-\frac{1}{p}\right) \int_{\mathbb{R}^{N}} \Lambda h(\varepsilon z) u_{+}^{q} d z \\
& <\left[\left(\frac{1}{2}-\frac{1}{p}\right)-\left(\frac{1}{q}-\frac{1}{p}\right) \frac{p-2}{p-q}\right]\|u\|_{H}^{2} \\
& =-\frac{(2-q)(p-2)}{2 p q}\|u\|_{H}^{2}<0 .
\end{aligned}
$$

By the definitions of $\alpha_{\varepsilon}$ and $\alpha_{\varepsilon}^{+}$, we deduce that $\alpha_{\varepsilon} \leq \alpha_{\varepsilon}^{+}<0$.

(ii) See the proof of Lemma 2.5 (iv).

Applying Ekeland's variational principle and using the same argument in Cao and Zhou [7] or Tarantello [11], we have the following lemma.

Lemma 2.8 (i) There exists a (PS $)_{\alpha_{\varepsilon}}$-sequence $\left\{u_{n}\right\}$ in $\mathbf{M}_{\varepsilon}$ for $J_{\varepsilon}$;

(ii) There exists a (PS $)_{\alpha_{\varepsilon}^{+}}$-sequence $\left\{u_{n}\right\}$ in $\mathbf{M}_{\varepsilon}^{+}$for $J_{\varepsilon}$;

(iii) There exists a $(P S)_{\alpha_{\varepsilon}^{-}-\text {sequence }}\left\{u_{n}\right\}$ in $\mathbf{M}_{\varepsilon}^{-}$for $J_{\varepsilon}$. 


\section{Existence of a ground state solution}

In order to prove the existence of positive solutions, we claim that $J_{\varepsilon}$ satisfies the (PS) $\beta_{\beta^{-}}$ condition in $H^{1}\left(\mathbb{R}^{N}\right)$ for $\beta \in\left(-\infty, \gamma_{\infty}-C_{0} \Lambda^{\frac{2}{2-q}}\right)$, where $\Lambda=\varepsilon^{2(p-q) /(p-2)}$ and $C_{0}$ is defined in the following lemma.

Lemma 3.1 Assume that $h$ satisfies $(h 1)$ and $0<\Lambda\left(=\varepsilon^{2(p-q) /(p-2)}\right)<\Lambda_{0}$. If $\left\{u_{n}\right\}$ is a $(P S)_{\beta}$-sequence in $H^{1}\left(\mathbb{R}^{N}\right)$ for $J_{\varepsilon}$ with $u_{n} \rightarrow u$ weakly in $H^{1}\left(\mathbb{R}^{N}\right)$, then $J_{\varepsilon}^{\prime}(u)=0$ in $H^{-1}$ $\left(\mathbb{R}^{N}\right)$ and $J_{\varepsilon}(u) \geq-C_{0} \Lambda^{\frac{2}{2-q}} \geq-C_{0}^{\prime}$, where

$$
\left.C_{0}=(2-q)\left[(p-q)\|h\|_{\#} S^{q}\right]^{\frac{2}{2-q}} / 2 p q(p-2)^{\frac{q}{2-q}}\right] \text {, }
$$

and

$$
C_{0}^{\prime}=\left[(p-2)(2-q)^{\frac{p}{p-2}}\right] /\left\{2 p q\left[f_{\max }(p-q)\right]^{\frac{2}{p-2}} \frac{2 p}{p-2}\right\} .
$$

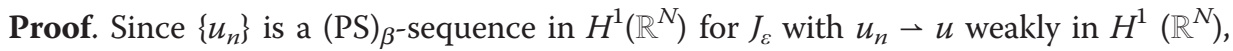
it is easy to check that $J_{\varepsilon}^{\prime}(u)=0$ in $H^{-1}\left(\mathbb{R}^{N}\right)$ and $u \geq 0$. Then we have $\left\langle J_{\varepsilon}^{\prime}(u), u\right\rangle=0$, that is, $\int_{\mathbb{R}^{N}} f(\varepsilon z) u^{p} d z=\|u\|_{H}^{2}-\int_{\mathbb{R}^{N}} \Lambda h(\varepsilon z) u^{q} d z$. Hence, by the Young inequality

$$
\begin{aligned}
\left(p_{1}=\frac{2}{q}\right. \text { and } & \left.p_{2}=\frac{2}{2-q}\right) \\
J_{\varepsilon}(u) & =\left(\frac{1}{2}-\frac{1}{p}\right)\|u\|_{H}^{2}-\left(\frac{1}{q}-\frac{1}{p}\right) \int_{\mathbb{R}^{N}} \Lambda h(\varepsilon z) u^{q} d z \\
& \geq \frac{p-2}{2 p}\|u\|_{H}^{2}-\frac{p-q}{p q} \Lambda\|h\|_{\#} S^{q}\|u\|_{H}^{q} \\
& \geq \frac{p-2}{2 p}\|u\|_{H}^{2}-\frac{p-2}{p q}\left[\frac{q\|u\|_{H}^{2}}{2}+\left(\frac{p-q}{p-2} \Lambda\|h\|_{\#} S^{q}\right)^{\frac{2}{2-q}} \frac{2-q}{2}\right] \\
& \geq-\frac{(p-2)(2-q)}{2 p q\left[f_{\max }(p-q)\right]^{\frac{2}{p-2}} \frac{2 p}{p-2}} .
\end{aligned}
$$

Lemma 3.2 Assume that $f$ and $h$ satisfy $(f 1)$ and (h1). If $0<\Lambda\left(=\varepsilon^{2(p-q) /(p-2)}\right)<\Lambda_{0}$, then $J_{\varepsilon}$ satisfies the $(P S)_{\beta}$-condition in $H^{1}\left(\mathbb{R}^{N}\right)$ for $\beta \in\left(-\infty, \gamma_{\infty}-C_{0} \frac{2}{2-q}\right)$. 
Proof. Let $\left\{u_{n}\right\}$ be a $(\mathrm{PS})_{\beta}$-sequence in $H^{1}\left(\mathbb{R}^{N}\right)$ for $J_{\varepsilon}$ such that $J_{\varepsilon}\left(u_{n}\right)=\beta+o_{n}(1)$ and $J_{\varepsilon}^{\prime}\left(u_{n}\right)=o_{n}(1)$ in $H^{-1}\left(\mathbb{R}^{N}\right)$. Then

$$
\begin{aligned}
|\beta|+c_{n}+\frac{d_{n}\left\|u_{n}\right\|_{H}}{p} & \geq J_{\varepsilon}\left(u_{n}\right)-\frac{1}{p}\left\langle J^{\prime}\left(u_{n}\right),\left(u_{n}\right)\right\rangle \\
& =\left(\frac{1}{2}-\frac{1}{p}\right)\left\|u_{n}\right\|_{H}^{2}-\left(\frac{1}{q}-\frac{1}{p}\right) \int_{\mathbb{R}^{N}} \frac{2(p-q)}{p-2} h(\varepsilon z)\left(u_{n}\right)_{+}^{q} d z \\
& \geq \frac{p-2}{2 p}\left\|u_{n}\right\|_{H}^{2}-\frac{p-q}{p q} \Lambda\|h\|_{\#} S^{q}\left\|u_{n}\right\|_{H}^{q},
\end{aligned}
$$

where $c_{n}=o_{n}(1), d_{n}=o_{n}(1)$ as $n \rightarrow \infty$. It follows that $\left\{u_{n}\right\}$ is bounded in $H^{1}\left(\mathbb{R}^{N}\right)$. Hence, there exist a subsequence $\left\{u_{n}\right\}$ and a nonnegative $u \in H^{1}\left(\mathbb{R}^{N}\right)$ such that $J_{\varepsilon}^{\prime}(u)=0$ in $H^{-1}\left(\mathbb{R}^{N}\right), u_{n} \rightarrow u$ weakly in $H^{1}\left(\mathbb{R}^{N}\right), u_{n} \rightarrow u$ a.e. in $\mathbb{R}^{N}, u_{n} \rightarrow u$ strongly in $L_{\text {loc }}^{s}\left(\mathbb{R}^{N}\right)$ for any $1 \leq s<2^{*}$. Using the Brézis-Lieb lemma to get (3.1) and (3.2) below.

$$
\begin{aligned}
& \int_{\mathbb{R}^{N}} f(\varepsilon z)\left(u_{n}-u\right)_{+}^{p} d z=\int_{\mathbb{R}^{N}} f(\varepsilon z)\left(u_{n}\right)_{+}^{p} d z-\int_{\mathbb{R}^{N}} f(\varepsilon z) u^{p} d z+o_{n}(1) ; \\
& \int_{\mathbb{R}^{N}} h(\varepsilon z)\left(u_{n}-u\right)_{+}^{q} d z=\int_{\mathbb{R}^{N}} h(\varepsilon z)\left(u_{n}\right)_{+}^{q} d z-\int_{\mathbb{R}^{N}} h(\varepsilon z) u^{q} d z+o_{n}(1) .
\end{aligned}
$$

Next, claim that

$$
\int_{\mathbb{R}^{N}} h(\varepsilon z)\left|u_{n}-u\right|^{q} d z \rightarrow 0 \text { as } n \rightarrow \infty .
$$

For any $\sigma>0$, there exists $r>0$ such that $\int_{\left[B^{N}(0 ; r)\right]^{c}} h(\varepsilon z) \frac{p}{p-q} d z<\sigma$. By the Hölder inequality and the Sobolev embedding theorem, we get

$$
\begin{aligned}
\left|\int_{\mathbb{R}^{N}} h(\varepsilon z)\right| u_{n}-\left.u\right|^{q} d z \mid & \leq \int_{B^{N}(0 ; r)} h(\varepsilon z)\left|u_{n}-u\right|^{q} d z \\
& +\int_{\left[B^{N}(0 ; r)\right]^{c}} h(\varepsilon z)\left|u_{n}-u\right|^{q} d z \\
& \leq\|h\|_{\#}\left(\int_{B^{N}(0 ; r)}\left|u_{n}-u\right|^{p} d z\right)^{q / p} \\
& +S^{q}\left(\int_{\left.B^{N}(0 ; r)\right]^{c}} h(\varepsilon z) \frac{p}{p-q} d z\right)^{\frac{p-q}{p}}\left\|u_{n}-u\right\|_{H}^{q} \\
& \leq C^{\prime} \sigma+o_{n}(1) . \\
& \left.\because\left\{u_{n}\right\} \text { is bounded in } H^{1}\left(\mathbb{R}^{N}\right) \text { and } u_{n} \rightarrow u \text { in } L_{l o c}^{p}\left(\mathbb{R}^{N}\right)\right)
\end{aligned}
$$


Applying $(f 1)$ and $u_{n} \rightarrow u$ in $L_{l o c}^{p}\left(\mathbb{R}^{N}\right)$, we get that

$$
\int_{\mathbb{R}^{N}} f(\varepsilon z)\left(u_{n}-u\right)_{+}^{p} d z=\int_{\mathbb{R}^{N}} f_{\infty}\left(u_{n}-u\right)_{+}^{p} d z+o_{n}(1) .
$$

Let $p_{n}=u_{n}-u$. Suppose $p_{n} \rightarrow 0$ strongly in $H^{1}\left(\mathbb{R}^{N}\right)$. By (3.1)-(3.4), we deduce that

$$
\begin{aligned}
\left\|p_{n}\right\|_{H}^{2} & =\left\|u_{n}\right\|_{H}^{2}-\|u\|_{H}^{2}+o_{n}(1) \\
& =\int_{\mathbb{R}^{N}} f(\varepsilon z)\left(u_{n}\right)_{+}^{p} d z-\int_{\mathbb{R}^{N}} \varepsilon \frac{2(p-q)}{p-2} h(\varepsilon z)\left(u_{n}\right)_{+}^{q} d z \\
& -\int_{\mathbb{R}^{N}} f(\varepsilon z) u^{p} d z+\int_{\mathbb{R}^{N}} \varepsilon \frac{2(p-q)}{p-2} h(\varepsilon z) u^{q} d z+o_{n}(1) \\
& =\int_{\mathbb{R}^{N}} f(\varepsilon z)\left(u_{n}-u\right)_{+}^{p} d z+o_{n}(1)=\int_{\mathbb{R}^{N}} f_{\infty}\left(p_{n}\right)_{+}^{p} d z+o_{n}(1) .
\end{aligned}
$$

Then

$$
\begin{aligned}
I_{\infty}\left(p_{n}\right) & =\frac{1}{2}\left\|p_{n}\right\|_{H}^{2}-\frac{1}{p} \int_{\mathbb{R}^{N}} f_{\infty}\left(p_{n}\right)_{+}^{p} d z \\
& =\left(\frac{1}{2}-\frac{1}{p}\right)\left\|p_{n}\right\|_{H}^{2}+o_{n}(1)>0 .
\end{aligned}
$$

By Theorem 4.3 in Wang [12], there exists a sequence $\left\{s_{n}\right\} \subset \mathbb{R}^{+}$such that $s_{n}=1+$ $o_{n}(1),\left\{s_{n} p_{n}\right\} \subset \mathbf{N}_{\infty}$ and $I_{\infty}\left(s_{n} p_{n}\right)=I_{\infty}\left(p_{n}\right)+o_{n}(1)$. It follows that

$$
\begin{aligned}
\gamma_{\infty} & \leq I_{\infty}\left(s_{n} p_{n}\right)=I_{\infty}\left(p_{n}\right)+o_{n}(1) \\
& =J_{\varepsilon}\left(u_{n}\right)-J_{\varepsilon}(u)+o_{n}(1) \\
& =\beta-J_{\varepsilon}(u)+o_{n}(1)<\gamma_{\infty},
\end{aligned}
$$

which is a contradiction. Hence, $u_{n} \rightarrow u$ strongly in $H^{1}\left(\mathbb{R}^{N}\right)$.

Remark 3.3 By Lemma 1.1, we obtain

$$
\begin{aligned}
& \qquad C_{0}^{\prime}=\frac{2-q}{q}\left(\frac{2-q}{p-q}\right)^{\frac{2}{p-2}} \gamma_{\max }<\gamma_{\max }<\gamma_{\infty}, \\
& \text { and } \gamma_{\infty}-C_{0} \Lambda^{\frac{2}{2-q}}>0 \text { for } 0<\Lambda<\Lambda_{0} .
\end{aligned}
$$

By Lemma $2.8(i)$, there is a (PS) $)_{\varepsilon}$-sequence $\left\{u_{n}\right\}$ in $\mathbf{M}_{\varepsilon}$ for $J_{\varepsilon}$. Then we prove that Equation $\left(E_{\varepsilon}\right)$ admits a positive ground state solution $u_{0}$ in $\mathbb{R}^{N}$.

Theorem 3.4 Under assumptions (f1), (h1), if $0<\Lambda\left(=\varepsilon^{2(p-q) /(p-2)}\right)<\Lambda_{0}$, then there exists at least one positive ground state solution $u_{0}$ of Equation $\left(E_{\varepsilon}\right)$ in $\mathbb{R}^{N}$. Moreover, we have that $u_{0} \in \mathbf{M}_{\varepsilon}^{+}$and

$$
J_{\varepsilon}\left(u_{0}\right)=\alpha_{\varepsilon}=\alpha_{\varepsilon}^{+} \geq-C_{0} \Lambda^{\frac{2}{2-q}} .
$$


Proof. By Lemma $2.8(i)$, there is a minimizing sequence $\left\{u_{n}\right\} \subset \mathbf{M}_{\varepsilon}$ for $J_{\varepsilon}$ such that $J_{\varepsilon}\left(u_{n}\right)=\alpha_{\varepsilon}+o_{n}(1)$ and $J_{\varepsilon}^{\prime}\left(u_{n}\right)=o_{n}(1)$ in $H^{-1}\left(\mathbb{R}^{N}\right)$. Since $\alpha_{\varepsilon}<0<\gamma^{\infty}-C_{0} \Lambda \frac{2}{2-q}$, by Lemma 3.2, there exist a subsequence $\left\{u_{n}\right\}$ and $u_{0} \in H^{1}\left(\mathbb{R}^{N}\right)$ such that $u_{n} \rightarrow u_{0}$ strongly in $H^{1}\left(\mathbb{R}^{N}\right)$. It is easy to see that $u_{0} \nsupseteq 0$ is a solution of Equation $\left(E_{\varepsilon}\right)$ in $\mathbb{R}^{N}$ and $J_{\varepsilon}\left(u_{0}\right)=\alpha_{\varepsilon}$. Next, we claim that $u_{0} \in \mathrm{M}_{\varepsilon}^{+}$. On the contrary, assume that $u_{0} \in \mathbf{M}_{\varepsilon}^{-}\left(\mathbf{M}_{\varepsilon}^{0}=\emptyset\right.$ for $\left.0<\Lambda\left(=\varepsilon^{2(p-q) /(p-2)}\right)<\Lambda_{0}\right)$.

We get that

$$
\int_{\mathbb{R}^{N}} \Lambda h(\varepsilon z)\left(u_{0}\right)_{+}^{q} d z>0 .
$$

Otherwise,

$$
\begin{aligned}
0 & =\int_{\mathbb{R}^{N}} \Lambda h(\varepsilon z)\left(u_{0}\right)_{+}^{q} d z=\int_{\mathbb{R}^{N}} \Lambda h(\varepsilon z)\left(u_{n}\right)_{+}^{q} d z+o_{n}(1) \\
& =\left\|u_{n}\right\|_{H}^{2}-\int_{\mathbb{R}^{N}} f(\varepsilon z)\left(u_{n}\right)_{+}^{p} d z+o_{n}(1) .
\end{aligned}
$$

It follows that

$$
\alpha_{\varepsilon}+o_{n}(1)=J_{\varepsilon}\left(u_{n}\right)=\left(\frac{1}{2}-\frac{1}{p}\right)\left\|u_{n}\right\|_{H}^{2}+o_{n}(1)
$$

which contradicts to $\alpha_{\varepsilon}<0$. By Lemma $2.6(i i)$, there exist positive numbers $t^{+}<\bar{t}<t^{-}=1$ such that $t^{+} u_{0} \in \mathbf{M}_{\varepsilon}^{+}, t^{-} u_{0} \in \mathbf{M}_{\varepsilon}^{-}$and

$$
J_{\varepsilon}\left(t^{+} u_{0}\right)<J_{\varepsilon}\left(t^{-} u_{0}\right)=J_{\varepsilon}\left(u_{0}\right)=\alpha_{\varepsilon},
$$

which is a contradiction. Hence, $u_{0} \in \mathbf{M}_{\varepsilon}^{+}$and

$$
-C_{0} \Lambda^{\frac{2}{2-q}} \leq J_{\varepsilon}\left(u_{0}\right)=\alpha_{\varepsilon}=\alpha_{\varepsilon}^{+} .
$$

By Lemma 2.4 and the maximum principle, then $u_{0}$ is a positive solution of Equation $\left(E_{\varepsilon}\right)$ in $\mathbb{R}^{N}$.

\section{Existence of $\boldsymbol{k}+1$ solutions}

From now, we assume that $f$ and $h$ satisfy $(f 1)-(f 2)$ and $(h 1)$. Let $w \in H^{1}\left(\mathbb{R}^{N}\right)$ be the unique, radially symmetric, and positive ground state solution of Equation $(E 0)$ in $\mathbb{R}^{N}$ for $f=f_{\max }$. Recall the facts (or see Bahri and Li [13], Bahri and Lions [14], Gidas et al. [15], and Kwong [16]).

(i) $w \in L^{\infty}\left(\mathbb{R}^{N}\right) \cap C_{l o c}^{2, \theta}\left(\mathbb{R}^{N}\right)$ for some $0<\theta<1$ and $\lim _{|z| \rightarrow \infty} w(z)=0$;

(ii) for any $\varepsilon>0$, there exist positive numbers $C_{1}, C_{1}, C_{2}^{\varepsilon}$, and $C_{3}^{\varepsilon}$ such that for all $z \in \mathbb{R}^{N}$

$$
C_{2}^{\varepsilon} \exp (-(1-\varepsilon)|z|) \leq w(z) \leq C_{1} \exp (-|z|)
$$

and

$$
|\nabla w(z)| \leq C_{3}^{\varepsilon} \exp (-(1-\varepsilon)|z| .)
$$

For $1 \leq i \leq k$, we define

$$
w_{\varepsilon}^{i}(z)=w\left(z-\frac{a^{i}}{\varepsilon}\right), \text { where } f\left(a^{i}\right)=f_{\max } .
$$


Clearly, $w_{\varepsilon}^{i}(z) \in H^{1}\left(\mathbb{R}^{N}\right)$. By Lemma $2.6(i i)$, there is a unique number $\left(t_{\varepsilon}^{i}\right)^{-}>0$ such that $\left(t_{\varepsilon}^{i}\right)^{-} w_{\varepsilon}^{i} \in \mathrm{M}_{\varepsilon}^{-} \subset \mathrm{M}_{\varepsilon}$, where $1 \leq i \leq k$.

We need to prove that

$$
\lim _{\varepsilon \rightarrow 0+} J_{\varepsilon}\left(\left(t_{\varepsilon}^{i}\right)^{-} w_{\varepsilon}^{i}\right) \leq \gamma_{\max } \text { uniformly in } i .
$$

Lemma 4.1 (i) There exists a number $t_{0}>0$ such that for $0 \leq t \leq t_{0}$ and any $\varepsilon>0$, we have that

$$
J_{\varepsilon}\left(t w_{\varepsilon}^{i}\right)<\gamma_{\max } \text { uniformly in } i ;
$$

(ii) There exist positive numbers $t_{1}$ and $\varepsilon_{1}$ such that for any $t>t_{1}$ and $\varepsilon<\varepsilon_{1}$, we have that $J_{\varepsilon}\left(t w_{\varepsilon}^{i}\right)<0$ uniformly in $i$.

Proof. (i) Since $J_{\varepsilon}$ is continuous in $H^{1}\left(\mathbb{R}^{N}\right),\left\{w_{\varepsilon}^{i}\right\}$ is uniformly bounded in $H^{1}\left(\mathbb{R}^{N}\right)$ for any $\varepsilon>0$, and $\gamma_{\max }>0$, there is $t_{0}>0$ such that for $0 \leq t \leq t_{0}$ and any $\varepsilon>0$

$$
J_{\varepsilon}\left(t w_{\varepsilon}^{i}\right)<\gamma_{\max } .
$$

(ii) There is an $r_{0}>0$ such that $f(z) \geq f_{\max } / 2$ for $z \in B^{N}\left(a^{i} ; r_{0}\right)$ uniformly in $i$. Then there exists $\varepsilon_{1}>0$ such that for $\varepsilon<\varepsilon_{1}$

$$
\begin{aligned}
J_{\varepsilon}\left(t w_{\varepsilon}^{i}\right) & =\frac{t^{2}}{2}\left\|w_{\varepsilon}^{i}\right\|_{H}^{2}-\frac{t^{p}}{p} \int_{\mathbb{R}^{N}} f(\varepsilon z)\left(w_{\varepsilon}^{i}\right)^{p} d z-\frac{t^{q}}{q} \int_{\mathbb{R}^{N}} \Lambda h(\varepsilon z)\left(w_{\varepsilon}^{i}\right)^{q} d z \\
& \leq \frac{t^{2}}{2} \int_{\mathbb{R}^{N}}\left[|\nabla w|^{2} w^{2}\right] d z-\frac{t^{p}}{2 p} \int_{B^{N}(0 ; 1)} f_{\max } w^{p} d z .
\end{aligned}
$$

Thus, there is $t_{1}>0$ such that for any $t>t_{1}$ and $\varepsilon<\varepsilon_{1}$

$$
J_{\varepsilon}\left(t w_{\varepsilon}^{i}\right)<0 \text { uniformly in } i .
$$

Lemma 4.2 Under assumptions (f1), (f2), and (h1). If $0<\Lambda\left(=\varepsilon^{2(p-q) /(p-2)}\right)<q \Lambda_{0} / 2$, then

$\lim _{\varepsilon \rightarrow 0+} \sup _{t \rightarrow 0} J_{\varepsilon}\left(t w_{\varepsilon}^{i}\right) \leq \gamma_{\max }$ uniformly in $i$.

Proof. By Lemma 4.1, we only need to show that

$$
\lim _{\varepsilon \rightarrow 0+} \sup _{t_{0} \leq t \leq t_{1}} J_{\varepsilon}\left(t w_{\varepsilon}^{i}\right) \leq \gamma_{\max } \text { uniformly in } i .
$$

We know that $\sup _{t \geq 0} I_{\max }(t w)=\gamma_{\max }$. For $t_{0} \leq t \leq t_{1}$, we get

$$
\begin{aligned}
J_{\varepsilon}\left(t w_{\varepsilon}^{i}\right) & =\frac{1}{2}\left\|t w_{\varepsilon}^{i}\right\|_{H}^{2}-\frac{1}{p} \int_{\mathbb{R}^{N}} f(\varepsilon z)\left(t w_{\varepsilon}^{i}\right)^{p} d z-\frac{1}{q} \int_{\mathbb{R}^{N}} \Lambda h(\varepsilon z)\left(t w_{\varepsilon}^{i}\right)^{q} d z \\
& =\frac{t^{2}}{2} \int_{\mathbb{R}^{N}}\left[\left|\nabla w\left(z-\frac{a^{i}}{\varepsilon}\right)\right|^{2}+w\left(z-\frac{a^{i}}{\varepsilon}\right)\right] d z \\
& -\frac{t^{p}}{p} \int_{\mathbb{R}^{N}} f(\varepsilon z) w\left(z-\frac{a^{i}}{\varepsilon}\right)^{p} d z-\frac{t^{q}}{q} \int_{\mathbb{R}^{N}} \Lambda h(\varepsilon z) w\left(z-\frac{a^{i}}{\varepsilon}\right)^{q} d z \\
& =\left\{\frac{t^{2}}{p} \int_{\mathbb{R}^{N}}\left[|\nabla w|^{2}+w^{2}\right] d z-\frac{t^{p}}{p} \int_{\mathbb{R}^{N}} f_{\max } w^{p} d z\right\} \\
& +\frac{t^{p}}{p} \int_{\mathbb{R}^{N}}\left(f_{\max }-f(\varepsilon z)\right) w\left(z-\frac{a^{i}}{\varepsilon}\right)^{p} d z-\frac{t^{q}}{q} \Lambda \int_{\mathbb{R}^{N}} h(\varepsilon z) w\left(z-\frac{a^{i}}{\varepsilon}\right)^{q} d z \\
& \leq \gamma_{\max }+\frac{t_{1}^{p}}{p} \int_{\mathbb{R}^{N}}\left(f_{\max }-f(\varepsilon z)\right) w\left(z-\frac{a^{i}}{\varepsilon}\right)^{p} d z-\frac{t_{0}^{q}}{q} \Lambda \int_{\mathbb{R}^{N}} h(\varepsilon z) w\left(z-\frac{a^{i}}{\varepsilon}\right) d z .
\end{aligned}
$$


Since

$$
\begin{aligned}
& \int_{\mathbb{R}^{N}}\left(f_{\max }-f(\varepsilon z)\right) w\left(z-\frac{a^{i}}{\varepsilon}\right)^{p} d z \\
& =\int_{\mathbb{R}^{N}}\left[f_{\max }-f\left(\varepsilon z+a^{i}\right)\right] w^{p} d z=o(1) \text { as } \varepsilon \rightarrow 0^{+} \text {uniformly in } i,
\end{aligned}
$$

and

$$
\Lambda \int_{\mathbb{R}^{N}} h(\varepsilon z) w\left(z-\frac{a^{i}}{\varepsilon}\right)^{q} d z \leq \varepsilon^{\frac{2(p-q)}{p-2}}\|h\|_{\#} S^{q}\|w\|_{H}^{q}=o(1) \text { as } \varepsilon \rightarrow 0^{+}
$$

then $\lim _{\varepsilon \rightarrow 0+} \sup _{t_{0} \leq t \leq t_{1}} J_{\varepsilon}\left(t w_{\varepsilon}^{i}\right) \leq \gamma_{\max }$, that is, $\lim _{\varepsilon \rightarrow 0_{+}} \sup _{t \geq 0} J_{\varepsilon}\left(t w_{\varepsilon}^{i}\right) \leq \gamma_{\max }$ uniformly in $i$.

Applying the results of Lemmas 2.6, 2.7(ii), and 4.2, we can deduce that

$$
0<d_{0} \leq \alpha_{\varepsilon}^{-} \leq \gamma_{\max }+o(1) \text { as } \varepsilon \rightarrow 0^{+} .
$$

Since $\gamma_{\max }<\gamma_{\infty}$, there exists $\varepsilon_{0}>0$ such that

$$
\gamma_{\max }<\gamma_{\infty}-C_{0} \Lambda^{\frac{2}{2-q}} \text { for any } \varepsilon<\varepsilon_{0} .
$$

Choosing $0<\rho_{0}<1$ such that

$$
\overline{B_{\rho 0}^{N}\left(a^{i}\right)} \cap \overline{B_{\rho 0}^{N}\left(a^{j}\right)}=\emptyset \text { for } i \neq j \text { and } 1 \leq i, j \leq k,
$$

where $\overline{B_{\rho 0}^{N}\left(a^{i}\right)}=\left\{z \in \mathbb{R}^{N}|| z-a^{i} \mid \leq \rho 0\right\}$ and $f\left(a^{i}\right)=f_{\max }$. Define $\mathbf{K}=\left\{a^{i} \mid 1 \leq i \leq k\right\}$ and $\mathbf{K}_{\rho_{0} / 2}=\cup_{i=1}^{k} \overline{B_{\rho_{0} / 2}^{N}\left(a^{i}\right)}$. Suppose $\cup_{i=1}^{k} \overline{B_{\rho_{0}}^{N}\left(a^{i}\right)} \subset B_{r_{0}}^{N}(0)$ for some $r_{0}>0$.

Let $Q_{\varepsilon}: H^{1}\left(\mathbb{R}^{N}\right) \backslash\{0\} \rightarrow \mathbb{R}^{N}$ be given by

$$
Q_{\varepsilon}(u)=\frac{\int_{\mathbb{R}^{N}} \chi(\varepsilon z)|u|^{p} d z}{\int_{\mathbb{R}^{N}}|u|^{p} d z}
$$

where $\chi: \mathbb{R}^{N} \rightarrow \mathbb{R}^{N}, \chi(z)=z$ for $|z| \leq r_{0}$ and $\chi(z)=r_{0} z /|z|$ for $|z|>r_{0}$.

Lemma 4.3 There exists $0<\varepsilon^{0} \leq \varepsilon_{0}$ such that if $\varepsilon<\varepsilon^{0}$, then $Q_{\varepsilon}\left(\left(t_{\varepsilon}^{i}\right)^{-} w_{\varepsilon}^{i}\right) \in \mathbf{K}_{\rho_{0} / 2}$ for each $1 \leq i \leq k$.

Proof. Since

$$
\begin{aligned}
Q_{\varepsilon}\left(\left(t_{\varepsilon}^{i}\right)^{-} w_{\varepsilon}^{i}\right) & =\frac{\int_{\mathbb{R}^{N}} \chi(\varepsilon z)\left|w\left(z-\frac{a^{i}}{\varepsilon}\right)\right|^{p} d z}{\int_{\mathbb{R}^{N}}\left|w\left(z-\frac{a^{i}}{\varepsilon}\right)\right|^{p} d z} \\
& =\frac{\int_{\mathbb{R}^{N}} \chi\left(\varepsilon z+a^{i}\right)|w(z)|^{p} d z}{\int_{\mathbb{R}^{N}}|w(z)|^{p} d z} \\
& \rightarrow a^{i} \text { as } \varepsilon \rightarrow 0^{+},
\end{aligned}
$$

there exists $\varepsilon^{0}>0$ such that

$$
Q_{\varepsilon}\left(\left(t_{\varepsilon}^{i}\right)^{-} w_{\varepsilon}^{i}\right) \in \mathbf{K}_{\rho_{0} / 2} \text { for any } \varepsilon<\varepsilon^{0} \text { and each } 1 \leq i \leq k
$$


Lemma 4.4 There exists a number $\bar{\delta}>0$ such that if $u \in \mathbf{N}_{\varepsilon}$ and $I_{\varepsilon}(u) \leq \gamma_{\max }+\bar{\delta}$,

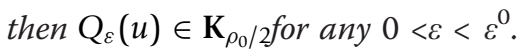

Proof. On the contrary, there exist the sequences $\left\{\varepsilon_{n}\right\} \subset \mathbb{R}^{+}$and $\left\{u_{n}\right\} \subset \mathbf{N}_{\varepsilon_{n}}$ such that $\varepsilon_{n} \rightarrow 0, I_{\varepsilon_{n}}\left(u_{n}\right)=\gamma_{\max }(>0)+o_{n}(1)$ as $n \rightarrow \infty$ and $Q_{\varepsilon_{n}}\left(u_{n}\right) \notin \mathbf{K}_{\rho_{0} / 2}$ for all $n \in \mathbb{N}$. It is easy to check that $\left\{u_{n}\right\}$ is bounded in $H^{1}\left(\mathbb{R}^{N}\right)$. Suppose $u_{n} \rightarrow 0$ strongly in $L^{p}\left(\mathbb{R}^{N}\right)$. Since

$$
\left\|u_{n}\right\|_{H}^{2}=\int_{\mathbb{R}^{N}} f\left(\varepsilon_{n} z\right)\left(u_{n}\right)_{+}^{p} d z \text { for each } n \in \mathbb{N},
$$

and

$$
I_{\varepsilon_{n}}\left(u_{n}\right)=\frac{1}{2}\left\|u_{n}\right\|_{H}^{2}-\frac{1}{p} \int_{\mathbb{R}^{N}} f\left(\varepsilon_{n} z\right)\left(u_{n}\right)_{+}^{p} d z=\gamma_{\max }+o_{n}(1),
$$

then

$$
\gamma_{\max }+o_{n}(1)=I_{\varepsilon_{n}}\left(u_{n}\right)=\left(\frac{1}{2}-\frac{1}{p}\right) \int_{\mathbb{R}^{N}} f\left(\varepsilon_{n} z\right)\left(u_{n}\right)_{+}^{p} d z=o_{n}(1),
$$

which is a contradiction. Thus, $u_{n} \rightarrow 0$ strongly in $L^{p}\left(\mathbb{R}^{N}\right)$. Applying the concentration-compactness principle (see Lions [17] or Wang [[12], Lemma 2.16]), then there exist a constant $d_{0}>0$ and a sequence $\left\{\widetilde{z_{n}}\right\} \subset \mathbb{R}^{N}$ such that

$$
\int_{B^{N}\left(\widetilde{z_{n}} ; 1\right)}\left|u_{n}(z)\right|^{2} d z \geq d_{0}>0 .
$$

Let $v_{n}(z)=u_{n}\left(z+\widetilde{z_{n}}\right)$, there are a subsequence $\left\{v_{n}\right\}$ and $v \in H^{1}\left(\mathbb{R}^{N}\right)$ such that $v_{n} \rightarrow v$ weakly in $H^{1}\left(\mathbb{R}^{N}\right)$. Using the similar computation in Lemma 2.6 , there is a sequence $\left\{s_{\text {max }}^{n}\right\} \subset \mathbb{R}^{+}$such that $\widetilde{v_{n}}=s_{\max }^{n} v_{n} \in \mathbf{N}_{\max }$ and

$$
\begin{aligned}
0 & <\gamma_{\max } \leq I_{\max }\left(\widetilde{v_{n}}\right) \leq I_{\varepsilon_{n}}\left(s_{\max }^{n} u_{n}\right) \\
& \leq I_{\varepsilon_{n}}\left(u_{n}\right)=\gamma_{\max }+o_{n}(1) \text { as } n \rightarrow \infty .
\end{aligned}
$$

We deduce that a convergent subsequence $\left\{s_{\max }^{n}\right\}$ satisfies $s_{\max }^{n} \rightarrow s_{0}>0$. Then there are subsequences $\left\{\tilde{v_{n}}\right\}$ and $\tilde{v} \in H^{1}\left(\mathbb{R}^{N}\right)$ such that $\tilde{v}_{n} \rightarrow \tilde{v}\left(=s_{0} v\right)$ weakly in $H^{1}\left(\mathbb{R}^{N}\right)$. By (4.2), then $\tilde{v} \neq 0$. Moreover, we can obtain that $\widetilde{v_{n}} \rightarrow \tilde{v}$ strongly in $H^{1}\left(\mathbb{R}^{N}\right)$ and $I_{\max }(\tilde{v})=\gamma_{\max }$. Now, we want to show that there exists a subsequence $\left\{z_{n}\right\}=\left\{\varepsilon_{n} \widetilde{z_{n}}\right\}$ such that $z_{n} \rightarrow z_{0} \in \mathbf{K}$.

(i) Claim that the sequence $\left\{z_{n}\right\}$ is bounded in $\mathbb{R}^{N}$. On the contrary, assume that $\left|z_{n}\right|$ $\rightarrow \infty$, then

$$
\begin{aligned}
\gamma_{\max } & =I_{\max }(\tilde{v})<I_{\infty}(\tilde{v}) \\
& \leq \liminf _{n \rightarrow \infty}\left[\frac{1}{2}\left\|\widetilde{v_{n}}\right\|_{H}^{2}-\frac{1}{p} \int_{\mathbb{R}^{N}} f\left(\varepsilon_{n} z+z_{n}\right)\left(\widetilde{v_{n}}\right)_{+}^{p} d z\right] \\
& =\liminf _{n \rightarrow \infty}\left[\frac{\left(s_{\max }^{n}\right)^{2}}{2}\left\|u_{n}\right\|_{H}^{2}-\frac{\left(s_{\max }^{n}\right)^{p}}{p} \int_{\mathbb{R}^{N}} f\left(\varepsilon_{n} z\right)\left(u_{n}\right)_{+}^{p} d z\right] \\
& =\liminf _{n \rightarrow \infty} I_{\varepsilon_{n}}\left(s_{\max }^{n} u_{n}\right) \leq \liminf _{n \rightarrow \infty} I_{\varepsilon_{n}}\left(u_{n}\right)=\gamma_{\max }
\end{aligned}
$$


which is a contradiction.

(ii) Claim that $z_{0} \in \mathbf{K}$. On the contrary, assume that $z_{0} \notin \mathbf{K}$, that is, $f\left(z_{0}\right)<f_{\max }$. Then using the above argument to obtain that

$$
\begin{aligned}
\gamma_{\max } & =I_{\max }(\tilde{v})<\frac{1}{2}\|\tilde{v}\|_{H}^{2}-\frac{1}{p} \int_{\mathbb{R}^{N}} f\left(z_{0}\right)(\tilde{v})_{+}^{p} d z \\
& \leq \liminf _{n \rightarrow \infty}\left[\frac{1}{2}\left\|\tilde{v}_{n}\right\|_{H}^{2}-\frac{1}{p} \int_{\mathbb{R}^{N}} f\left(\varepsilon_{n} z+z_{n}\right)\left(\tilde{v_{n}}\right)_{+}^{p} d z\right] \\
& =\gamma_{\max }
\end{aligned}
$$

which is a contradiction. Since $v_{n} \rightarrow v \neq 0$ in $H^{1}\left(\mathbb{R}^{N}\right)$, we have that

$$
\begin{aligned}
Q_{\varepsilon_{n}}\left(u_{n}\right) & =\frac{\int_{\mathbb{R}^{N}} \chi\left(\varepsilon_{n} z\right)\left|v_{n}\left(z-\widetilde{z_{n}}\right)\right|^{p} d z}{\int_{\mathbb{R}^{N}}\left|v_{n}\left(z-\widetilde{z_{n}}\right)\right|^{p} d z} \\
& =\frac{\int_{\mathbb{R}^{N}} \chi\left(\varepsilon_{n} z+\varepsilon_{n} \widetilde{z_{n}}\right)\left|v_{n}\right|^{p} d z}{\int_{\mathbb{R}^{N}}\left|v_{n}\right|^{p} d z} \rightarrow z_{0} \subset \mathbf{K}_{\rho_{0} / 2} \text { as } n \rightarrow \infty,
\end{aligned}
$$

which is a contradiction.

Hence, there exists a number $\bar{\delta}>0$ such that if $u \in \mathbf{N}_{\varepsilon}$ and $I_{\varepsilon}(u) \leq \gamma_{\max }+\bar{\delta}$, then $Q_{\varepsilon}(u) \in \mathbf{K}_{\rho_{0} / 2}$ for any $0<\varepsilon<\varepsilon^{0}$.

From (4.1), choosing $0<\delta_{0}<\bar{\delta}$ such that

$$
\gamma_{\max }+\delta_{0}<\gamma_{\infty}-C_{0} \Lambda^{\frac{2}{2-q}} \text { for any } 0<\varepsilon<\varepsilon^{0} .
$$

For each $1 \leq i \leq k$, define

$$
\begin{array}{r}
O_{\varepsilon}^{i}=\left\{u \in \mathbf{M}_{\varepsilon}^{-}|| Q_{\varepsilon}(u)-a^{i} \mid<\rho_{0}\right\}, \\
\partial O_{\varepsilon}^{i}=\left\{u \in \mathbf{M}_{\varepsilon}^{-}|| Q_{\varepsilon}(u)-a^{i} \mid=\rho_{0}\right\}, \\
\beta_{\varepsilon}^{i}=\inf _{u \in O_{\varepsilon}^{i}} J_{\varepsilon}(u) \text { and } \tilde{\beta}_{\varepsilon}^{i}=\inf _{u \in \partial O_{\varepsilon}^{i}} J_{\varepsilon}(u) .
\end{array}
$$

Lemma 4.5 If $u \in \mathrm{M}_{\varepsilon}^{-}$and $J_{\varepsilon}(u) \leq \gamma_{\max }+\delta_{0} / 2$, then there exists a number

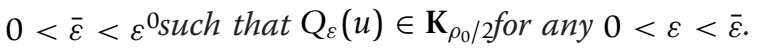

Proof. We use the similar computation in Lemma 2.6 to get that there is a unique positive number

$$
s_{\varepsilon}^{u}=\left(\frac{\|u\|_{H}^{2}}{\int_{\mathbb{R}^{N}} f(\varepsilon z) u_{+}^{p} d z}\right)^{1 /(p-2)}
$$

such that $s_{\varepsilon}^{u} u \in \mathbf{N}_{\varepsilon}$. We want to show that $s_{\varepsilon}^{u}<c$ for some constant $c>0$ (independent of $u$ ). First, since $u \in \mathbf{M}_{\varepsilon}^{-} \subset \mathbf{M}_{\varepsilon}$,

$$
0<d_{0} \leq \alpha_{\varepsilon}^{-} \leq J_{\varepsilon}(u) \leq \gamma_{\max }+\delta_{0} / 2,
$$

and $J_{\varepsilon}$ is coercive on $\mathbf{M}_{\varepsilon}$, then $0<c_{2}<\|u\|_{H}^{2}<c_{1}$ for some constants $c_{1}$ and $c_{2}$ (independent of $u$ ). Next, we claim that $\|u\|_{L^{p}}^{p}>c_{3}>0$ for some constant $c_{3}>0$ 
(independent of $u$ ). On the contrary, there exists a sequence $\left\{u_{n}\right\} \subset \mathbf{M}_{\varepsilon}^{-}$such that

$$
\left\|u_{n}\right\|_{L^{p}}^{p}=o_{n}(1) \text { as } n \rightarrow \infty .
$$

By (2.3),

$$
\frac{2-q}{p-q}<\frac{\int_{\mathbb{R}^{N}} f(\varepsilon z)\left(u_{n}\right)_{+}^{p} d z}{\left\|u_{n}\right\|_{H}^{2}} \leq \frac{f_{\max }\left\|u_{n}\right\|_{L^{p}}^{p}}{c_{2}}=o_{n}(1),
$$

which is a contradiction. Thus, $s_{\varepsilon}^{u}<c$ for some constant $c>0$ (independent of $u$ ). Now, we get that

$$
\begin{aligned}
\gamma_{\max }+\delta_{0} / 2 & \geq J_{\varepsilon}(u)=\sup _{t \geq 0} J_{\varepsilon}(t u) \geq J_{\varepsilon}\left(s_{\varepsilon}^{u} u\right) \\
& =\frac{1}{2}\left\|s_{\varepsilon}^{u} u\right\|_{H}^{2}-\frac{1}{p} \int_{\mathbb{R}^{N}} f(\varepsilon z)\left(s_{\varepsilon}^{u} u\right)_{+}^{p} d z-\frac{1}{q} \int_{\mathbb{R}^{N}} \Lambda h(\varepsilon z)\left(s_{\varepsilon}^{u} u\right)_{+}^{q} d z \\
& \geq I_{\varepsilon}\left(s_{\varepsilon}^{u} u\right)-\frac{1}{q} \int_{\mathbb{R}^{N}} \Lambda h(\varepsilon z)\left(s_{\varepsilon}^{u} u\right)_{+}^{q} d z .
\end{aligned}
$$

From the above inequality, we deduce that

$$
\begin{aligned}
I_{\varepsilon}\left(s_{\varepsilon}^{u} u\right) & \leq \gamma_{\max }+\delta_{0} / 2+\frac{1}{q} \int_{\mathbb{R}^{N}} \Lambda h(\varepsilon z)\left(s_{\varepsilon}^{u} u\right)_{+}^{q} d z \\
& \leq \gamma_{\max }+\delta_{0} / 2+\Lambda\|h\|_{\#} S^{q}\left\|s_{\varepsilon}^{u} u\right\|_{H}^{q} \\
& <\gamma_{\max }+\delta_{0} / 2+\Lambda c^{q}\left(c_{1}\right)^{q / 2}\|h\|_{\#} S^{q}, \text { where } \Lambda=\varepsilon^{2(p-q) /(p-2)} .
\end{aligned}
$$

Hence, there exists $0<\bar{\varepsilon}<\varepsilon^{0}$ such that for $0<\varepsilon<\bar{\varepsilon}$

$$
I_{\varepsilon}\left(s_{\varepsilon}^{u} u\right) \leq \gamma_{\max }+\delta_{0}, \text { where } s_{\varepsilon}^{u} u \in \mathbf{N}_{\varepsilon} .
$$

By Lemma 4.4, we obtain

$$
Q_{\varepsilon}\left(s_{\varepsilon}^{u} u\right)=\frac{\int_{\mathbb{R}^{N}} \chi(\varepsilon z)\left|s_{\varepsilon}^{u} u(z)\right|^{p} d z}{\int_{\mathbb{R}^{N}}\left|s_{\varepsilon}^{u} u(z)\right|^{p} d z} \in \mathbf{K}_{\rho_{0} / 2} \text { for any } 0<\varepsilon<\bar{\varepsilon},
$$

or $Q_{\varepsilon}(u) \in \mathbf{K}_{\rho_{0} / 2}$ for any $0<\varepsilon<\bar{\varepsilon}$.

Applying the above lemma, we get that

$$
\tilde{\beta}_{\varepsilon}^{i} \geq \gamma_{\max }+\delta_{0} / 2 \text { for any } 0<\varepsilon<\bar{\varepsilon} .
$$

By Lemmas 4.2, 4.3, and Equation (4.3), there exists $0<\varepsilon^{*} \leq \bar{\varepsilon}$ such that

$$
\beta_{\varepsilon}^{i} \leq J_{\varepsilon}\left(\left(t_{\varepsilon}^{i}\right)^{-} w_{\varepsilon}^{i}\right) \leq \gamma_{\max }+\delta_{0} / 3<\gamma_{\infty}-C_{0} \Lambda^{\frac{2}{2-q}} \text { for any } 0<\varepsilon<\varepsilon^{*} .
$$

Lemma 4.6 Given $u \in O_{\mathcal{\varepsilon}}^{i}$, then there exist an $\eta>0$ and a differentiable functional $l$ : $B(0 ; \eta) \subset H^{1}\left(\mathbb{R}^{N}\right) \rightarrow \mathbb{R}^{+}$such that $l(0)=1, l(v)(u-v) \in O_{\varepsilon}^{i}$ for any $v \in B(0 ; \eta)$ and

$$
\left.\left\langle l^{\prime}(v), \phi\right\rangle\right|_{(l, v)=(1,0)}=\frac{\left\langle\psi_{\varepsilon}^{\prime}(u), \phi\right\rangle}{\left\langle\psi_{\varepsilon}^{\prime}(u), u\right\rangle} \text { for any } \phi \in C_{c}^{\infty}\left(\mathbb{R}^{N}\right),
$$

where $\psi_{\varepsilon}(u)=\left\langle J_{\varepsilon}^{\prime}(u), u\right\rangle$. 
Proof. See Cao and Zhou [7].

Lemma 4.7 For each $1 \leq i \leq k$, there is a $(P S)_{\beta_{\varepsilon}^{i} \text { sequence }}\left\{u_{n}\right\} \subset O_{\varepsilon}^{i}$ in $H^{1}\left(\mathbb{R}^{N}\right)$ for $J_{\varepsilon}$.

Proof. For each $1 \leq i \leq k$, by (4.4) and (4.5),

$$
\beta_{\varepsilon}^{i}<\tilde{\beta}_{\varepsilon}^{i} \text { for any } 0<\varepsilon<\varepsilon^{*} .
$$

Then

$$
\beta_{\varepsilon}^{i}=\inf _{u \in O_{\varepsilon}^{i} \cup \partial O_{\varepsilon}^{i}} J_{\varepsilon}(u) \text { for any } 0<\varepsilon<\varepsilon^{*} .
$$

Let $\left\{u_{n}^{i}\right\} \subset O_{\varepsilon}^{i} \cup \partial O_{\varepsilon}^{i}$ be a minimizing sequence for $\beta_{\varepsilon}^{i}$. Applying Ekeland's variational principle, there exists a subsequence $\left\{u_{n}^{i}\right\}$ such that $J_{\varepsilon}\left(u_{n}^{i}\right)=\beta_{\varepsilon}^{i}+1 / n$ and

$$
J_{\varepsilon}\left(u_{n}^{i}\right) \leq J_{\varepsilon}(w)+\left\|w-u_{n}^{i}\right\|_{H} / n \text { for all } w \in O_{\varepsilon}^{i} \cup \partial O_{\varepsilon}^{i} .
$$

Using (4.7), we may assume that $u_{n}^{i} \in O_{\varepsilon}^{i}$ for sufficiently large $n$. By Lemma 4.6, then there exist an $\eta_{n}^{i}>0$ and a differentiable functional $l_{n}^{i}: B\left(0 ; \eta_{n}^{i}\right) \subset H^{1}\left(\mathbb{R}^{N}\right) \rightarrow \mathbb{R}^{+}$such that $l_{n}^{i}(0)=1$, and $l_{n}^{i}(v)\left(u_{n}^{i}-v\right) \in O_{\varepsilon}^{i}$ for $v \in B\left(0 ; \eta_{n}^{i}\right)$. Let $v_{\sigma}=\sigma v$ with $\|v\|_{H}=1$ and $0<\sigma<\eta_{n}^{i}$. Then $v_{\sigma} \in B\left(0, \eta_{n}^{i}\right)$ and $w_{\sigma}=l_{n}^{i}\left(v_{\sigma}\right)\left(u_{n}^{i}-v_{\sigma}\right) \in O_{\varepsilon^{\prime}}^{i}$. From (4.8) and by the mean value theorem, we get that as $\sigma \rightarrow 0$

$$
\begin{aligned}
& \frac{\left\|w_{\sigma}-u_{n}^{i}\right\|_{H} \geq \geq}{n} \geq J_{\varepsilon}\left(u_{n}^{i}\right)-J_{\varepsilon}\left(w_{\sigma}\right) \\
&=\left\langle J^{\prime}{ }_{\varepsilon}\left(t_{0} u_{n}^{i}+\left(1-t_{0}\right) w_{\sigma}\right), u_{n}^{i}-w_{\sigma}\right\rangle \text { where } t_{0} \in(0,1) \\
&=\left\langle J^{\prime}\left(u_{n}^{i}\right), u_{n}^{i}-w_{\sigma}\right\rangle+o\left(\left\|u_{n}^{i}-w_{\sigma}\right\|_{H}\right)\left(\because J_{\varepsilon} \in C^{1}\right) \\
&=\sigma l_{n}^{i}\left(v_{\sigma}\right)\left\langle J^{\prime}{ }_{\varepsilon}\left(u_{n}^{i}\right), v\right\rangle+\left(1-l_{n}^{i}\left(v_{\sigma}\right)\right)\left\langle J_{\varepsilon}^{\prime}\left(u_{n}^{i}\right), u_{n}^{i}\right\rangle+o\left(\left\|u_{n}^{i}-w_{\sigma}\right\|_{H}\right) \\
& \quad\left(\because l_{n}^{i}\left(v_{\sigma}\right) \rightarrow l_{n}^{i}(0)=1 \text { as } \sigma \rightarrow 0\right) \\
&= \sigma l_{n}^{i}(\sigma v)\left\langle J^{\prime}\left(u_{n}^{i}\right), v\right\rangle+o\left(\left\|u_{n}^{i}-w_{\sigma}\right\|_{H}\right) .
\end{aligned}
$$

Hence,

$$
\begin{aligned}
\left|\left\langle J^{\prime}\left(u_{n}^{i}\right), v\right\rangle\right| & \leq \frac{\left\|w_{\sigma}-u_{n}^{i}\right\|_{H}\left(\frac{1}{n}+|o(1)|\right)}{\sigma\left|l_{n}^{i}(\sigma v)\right|} \\
& \leq \frac{\left\|u_{n}^{i}\left(l_{n}^{i}(\sigma v)-l_{n}^{i}(0)\right)-\sigma v l_{n}^{i}(\sigma v)\right\|_{H}\left(\frac{1}{n}+|0(1)|\right)}{\sigma\left|l_{n}^{i}(\sigma v)\right|} \\
& \leq \frac{\left\|u_{n}^{i}\right\|_{H}\left|l_{n}^{i}(\sigma v)-l_{n}^{i}(0)\right|+\sigma\|v\|_{H}\left|l_{n}^{i}(\sigma v)\right|}{\sigma\left|l_{n}^{i}(\sigma v)\right|}\left(\frac{1}{n}+|o(1)|\right) \\
& \leq C\left(1+\left\|\left(l_{n}^{i}\right)^{\prime}(0)\right\|\right)\left(\frac{1}{n}+|o(1)|\right) .
\end{aligned}
$$

Since we can deduce that $\left\|\left(l_{n}^{i}\right)^{\prime}(0)\right\| \leq c$ for all $n$ and $i$ from (4.6), then $J_{\varepsilon}^{\prime}\left(u_{n}^{i}\right)=o_{n}(1)$ strongly in $H^{-1}\left(\mathbb{R}^{N}\right)$ as $n \rightarrow \infty$.

Theorem 4.8 Under assumptions $(f 1),\left(f_{2}\right)$, and $(h 1)$, there exists a positive number $\lambda^{*}$ $\left(\lambda^{*}=\left(\varepsilon^{*}\right)^{-2}\right)$ such that for $\lambda>\lambda^{*}$, Equation $\left(E_{\lambda}\right)$ has $k+1$ positive solutions in $\mathbb{R}^{N}$.

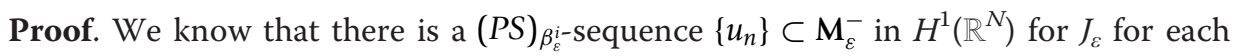

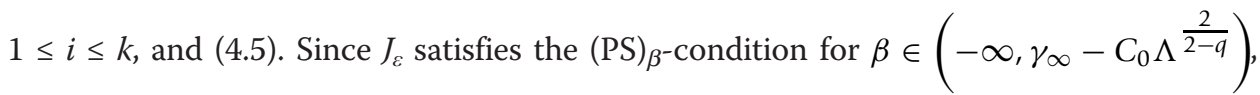
then $J_{\varepsilon}$ has at least $k$ critical points in $\mathbf{M}_{\varepsilon}^{-}$for $0<\varepsilon<\varepsilon^{*}$. It follows that Equation $\left(E_{\lambda}\right)$ 
has $k$ nonnegative solutions in $\mathbb{R}^{N}$. Applying the maximum principle and Theorem 3.4 , Equation $\left(E_{\lambda}\right)$ has $k+1$ positive solutions in $\mathbb{R}^{N}$.

\section{Acknowledgements}

The author was grateful for the referee's helpful suggestions and comments.

\section{Competing interests}

The author declares that he has no competing interests.

Received: 13 July 2011 Accepted: 24 February 2012 Published: 24 February 2012

\section{References}

1. Ambrosetti, A, Brezis, H, Cerami, G: Combined effects of concave and convex nonlinearities in some elliptic problems. J Funct Anal. 122, 519-543 (1994). doi:10.1006/jfan.1994.1078

2. $\mathrm{Wu}, \mathrm{TF}$ : On semilinear elliptic equations involving concave-convex non-linearities and sign-changing weight function. J Math Anal Appl. 318, 253-270 (2006). doi:10.1016/j.jmaa.2005.05.057

3. Ambrosetti, A, Garcia Azorero, J, Peral Alonso, I: Multiplicity results for some nonlinear elliptic equations. J Funct Anal. 137, 219-242 (1996). doi:10.1006/jfan.1996.0045

4. Brown, KJ, Zhang, Y: The Nehari manifold for a semilinear elliptic equation with a sign-changing weight function. J Diff Equ. 193, 481-499 (2003). doi:10.1016/S0022-0396(03)00121-9

5. de Figueiredo, DG, Gossez, JP, Ubilla, P: Local superlinearity and sub-linearity for indefinite semilinear elliptic problems. J Funct Anal. 199, 452-467 (2003). doi:10.1016/S0022-1236(02)00060-5

6. Zhu, XP: A perturbation result on positive entire solutions of a semilinear elliptic equation. J Diff Equ. 92, 163-178 (1991). doi:10.1016/0022-0396(91)90045-B

7. Cao, DM, Zhou, HS: Multiple positive solutions of nonhomogeneous semi-linear elliptic equations in $\mathbb{V}^{N}$. Proc Roy Soc Edinburgh, Sect A. 126, 443-463 (1996). doi:10.1017/S0308210500022836

8. Hirano, N: Existence of entire positive solutions for nonhomogeneous elliptic equations. Nonlinear Anal. 29, 889-901 (1997). doi:10.1016/S0362-546X(96)00176-9

9. Adachi, S, Tanaka, K: Four positive solutions for the semilinear elliptic equation: $-\Delta u+u=a(x) u^{p}+f(x)$ in $\nabla^{N}$. Calc $\operatorname{Var}$ Partial Diff Equ. 11, 63-95 (2000). doi:10.1007/s005260050003

10. Hsu, TS, Lin, HL: Four positive solutions of semilinear elliptic equations involving concave and convex nonlinearities in $\bigotimes^{N}$. J Math Anal Appl. 365, 758-775 (2010). doi:10.1016/j.jmaa.2009.12.004

11. Tarantello, G: On nonhomogeneous elliptic involving critical Sobolev exponent. Ann Inst H Poincaré Anal Non Linéaire. 9, 281-304 (1992)

12. Wang, HC: Palais-Smale approaches to semilinear elliptic equations in unbounded domains. Electron J Diff Equ Monogragh 142 (2004). 06

13. Bahri, $A, L i, Y Y$ : On a min-max procedure for the existence of a positive solution for certain scalar field equations in $\mathbb{}^{N}$. Rev Mat Iberoamericana. 6, 1-15 (1990)

14. Bahri, A, Lions, PL: On the existence of a positive solution of semilin-ear elliptic equations in unbounded domains. Ann Inst H Poincaré Anal Nonlinéaire. 14, 365-413 (1997)

15. Gidas, B, Ni, WM, Nirenberg, L: Symmetry and related properties via the maximum principle. Comm Math Phys. 68 , 209-243 (1979). doi:10.1007/BF01221125

16. Kwong, MK: Uniqueness of positive solutions of $\Delta u-u+u^{p}=0$ in $\bigotimes^{N}$. Arch Ration Mech Anal. 105, $234-266$ (1989)

17. Lions, PL: The concentration-compactness principle in the calculus of variations. The locally compact case. I II Ann Inst H Poincaré Anal Non-linéaire 1, 109-145 (1984). 223-283

doi:10.1186/1687-2770-2012-24

Cite this article as: Lin: Multiple positive solutions of semilinear elliptic equations involving concave and convex nonlinearities in $\mathbb{R}^{N}$. Boundary Value Problems 2012 2012:24.

\section{Submit your manuscript to a SpringerOpen ${ }^{\circ}$ journal and benefit from:}

- Convenient online submission

Rigorous peer review

- Immediate publication on acceptance

- Open access: articles freely available online

- High visibility within the field

- Retaining the copyright to your article

Submit your next manuscript at $>$ springeropen.com 